Proceedings the 44th International School and Conference on the Physics of Semiconductors "Jaszowiec 2015", Wisła 2015

\title{
Effect of Dielectric Medium Anisotropy on the Polarization Degree of Emission from a Single Quantum Dash
}

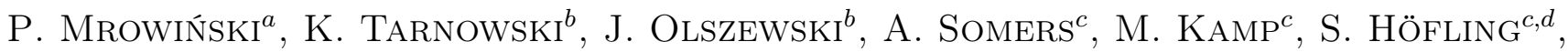 \\ J.P. Reithmaier ${ }^{e}$, W. UrbańCZyK ${ }^{b}$, J. Misiewicz ${ }^{a}$, P. MAchnikowski ${ }^{f}$ And G. SęK ${ }^{a}$ \\ ${ }^{a}$ Laboratory for Optical Spectroscopy of Nanostructures, Department of Experimental Physics, \\ Wrocław University of Technology, Wybrzeże Wyspiańskiego 27, 50-370 Wrocław, Poland \\ ${ }^{b}$ Department of Optics and Photonics, Wrocław University of Technology, Wrocław, Poland \\ ${ }^{c}$ Technische Physik, W.C. Röntgen-Center for Complex Material Systems, Universität Würzburg, Germany \\ ${ }^{d}$ SUPA, School of Physics and Astronomy, University of St. Andrews, North Haugh, \\ KY16 9SS St. Andrews, United Kingdom \\ ${ }^{e}$ Institute of Nanostructure Technologies and Analytics (INA), CINSaT, University of Kassel, \\ Heinrich-Plett-Str. 40, 34132 Kassel, Germany \\ ${ }^{f}$ Department of Theoretical Physics, Wrocław University of Technology, Wrocław, Poland
}

\begin{abstract}
Excitonic emission from single InAs/InGaAlAs/InP quantum dashes has been investigated in the context of degree of linear polarization by post-growth modification of its surrounding dielectric medium. We present optical spectroscopy measurements on a symmetric squared pedestal structures (mesas), and asymmetric rectangular ones oriented parallel or perpendicular to the main in-plane axis of the dashes [1-10]. Polarization resolved microphotoluminescence shows a significant quantitative modification of the degree of linear polarization value from $-20 \%$ up to $70 \%$. These results have been confronted with calculations of the coupling between the exciton transition dipole moment and electromagnetic field distributed in the vicinity of a quantum dash inside a processed mesa.
\end{abstract}

DOI: 10.12693/APhysPolA.129.A-48

PACS: 78.67.Hc, 71.35.-y, 78.55.-m

\section{Introduction}

In quantum information processing applications like linear optical quantum computing [1] or quantum key distribution (QKD) [2] it is fundamentally important to initialize and manipulate quantum superposition of photon states. In the most practical proposal for QKD, the so called BB84 protocol [3], unpolarized light pulses are randomly projected onto polarization selective elements to generate a cryptographic key. Such a scheme suffers from unavoidable losses, since randomly generated photons reach the transmission channel with only $50 \%$ chance. Moreover, attenuated laser pulses retain a certain nonzero probability of multiphoton events which reduces the security of the transfer $[2,4]$. Regarding these limitations a true single photon emitter with directly defined polarization state, on-demand operation and sub-Poissonian statistics is highly demanded. Another issue for practical devices is to provide a long-distance transmission channel with minimized losses which can be achieved by adjusting the photon wavelength to match the silica-fiberbased telecommunication windows at $1.3 \mu \mathrm{m}$ or $1.55 \mu \mathrm{m}$. All of these stimulates enormous effort to study robust single semiconductor quantum dots revealing their promising optical properties that can serve as a platform for single photon emitters [5-8] fulfilling the abovementioned requirements. However, to achieve a simultaneous strong suppression of multiphoton events, high collection efficiency, telecom spectral range and polarization control remains still a pending issue.

Hereby, we focus on the control of the degree of linear polarization (DOLP) which can be achieved by embedding a quantum-dot-like emitter inside asymmetric pedestals (in a shape of rectangular parallelepipeds), leading to a modification of the coupling between quantum emitter and optical field. Similar modifications of the coupling have already been demonstrated using symmetric pyramids [9], nanowires [10-12], ridge waveguide [13] or by resonant coupling to the electromagnetic field of micro- or nanocavity resonators [14-16]. In case of microcavities, the DOLP can be increased by tuning the emitter into resonance with one of the orthogonally polarized fundamental cavity modes [15]. Despite the high efficiency of such an approach, it requires a high quality microcavity structure which is realistic but still a technologically challenging task. Therefore, a much easier and more robust method is to pattern a dielectric medium of certain geometry, containing the single emitter inside. Up to now, the existing reports concern almost exclusively the attempts on the polarization control in the spectral range below $1 \mu \mathrm{m}$, with only a single report on modifying the QD emission polarization at the 3rd telecommunication window by tailoring a ridge waveguide structure [17]. 


\section{Experimental}

In this communication we present optical properties of excitons confined in single semiconductor InAs/InGaAlAs/InP quantum dashes (QDashes) which have exhibited antibunching at telecommunication wavelengths [8], significant fine structure splitting with nonzero degree of linear polarization [18] which enables the generation of single photons in a superposition of bright states resulting in linearly polarized spectral lines, and the ability to cancel the excitonic fine structure splitting by a magnetic field [19]. A sample with a layer of InAs QDashes was grown in an EIKO gas source molecularbeam epitaxy system in the Stransky-Krastanow mode by depositing $1.3 \mathrm{~nm}$ InAs (nominal thickness) at a sample temperature of $470^{\circ} \mathrm{C}$. The QDashes were embedded between two $\operatorname{In}_{0.53} \mathrm{Ga}_{0.23} \mathrm{Al}_{0.24} \mathrm{As}$ barriers, lattice matched to the $\operatorname{InP}(001)$ substrate. The entire structure is terminated with a $10 \mathrm{~nm}$ thick layer of InP. The QDash morphology shows a triangular-like shape in cross-section, with a base width of about $20 \mathrm{~nm}$ and a height of $3.5 \mathrm{~nm}$, whereas the length is estimated to be between 50 to hundreds of nanometers [20]. In order to post-select optically a single nanostructure from an inhomogeneous ensemble, electron beam lithography combined with wet chemical etching has been used to pattern mesa structures, since the areal density of nanostructures can exceed $5 \times 10^{10} \mathrm{~cm}^{-2}$. The processed mesas combined with a strong inhomogeneity of the ensemble enables to resolve optical transitions in single dashes for the used etched areas down to the size of $0.25 \mu \mathrm{m}^{2}$ $\left(600 \times 300 \mathrm{~nm}^{2}\right)$ [21]. The DOLP is tailored by using parallel and perpendicular orientation of rectangular mesas (aspect ratio 2:1) with respect to the QDash elongation axis, namely [1-10] crystallographic direction in this case, and squared mesas treated as a reference.

We performed microphotoluminescence $(\mu \mathrm{PL})$ experiments in a continuous-flow liquid-helium cryostat at temperature of approximately $5 \mathrm{~K}$. The spatial resolution is determined by the etched mesa size. In order to probe only a single mesa at a time (mesas are separated by $30 \mu \mathrm{m}$ ) and to efficiently collect the emitted radiation, we used a long working distance microscope objective with numerical aperture of NA $=0.4$. This corresponds to an approximately $2 \mu \mathrm{m}$ diffraction limited diameter of the excitation spot for continuous-wave semiconductor laser diode at $660 \mathrm{~nm}$. A spectral resolution below $30 \mu \mathrm{eV}$ has been obtained by a one-meter focal length monochromator, whereas the emission intensity of single spectral lines is integrated by a liquid nitrogen-cooled InGaAs linear detector. We focused on the ground state excitons predefined by approximately linear intensity dependence on the excitation power density and usually a noticeable fine structure splitting in the polarization resolved experiment [22]. For DOLP extraction we measured microphotoluminescence passing through a half-wave plate mounted in front of a linear polarizer and we performed a full 360 degree scan by rotating the birefringent element. The DOLP was calculated using a standard formula: DOLP $=\left(I_{H}+I_{V}\right) /\left(I_{H}-I_{V}\right)$, where $I_{H}, I_{V}$ represents emission polarized along [1-10] and [110] directions, respectively.

\section{Results and discussion}

In Fig. 1 we present photoluminescence spectra from a QDash ensemble with the maximum intensity at about $1530 \mathrm{~nm}$ detected for the orthogonal linear polarizations, and measured in the same way a single exciton emission from a rectangular submicrometer mesa structure oriented along the elongation direction of the dash. The excitation power density, similar in both cases, has been kept low to ensure the excitonic ground state origin of DOLP for a reliable comparison. In the first case, we attribute the $25 \%$ DOLP to the intrinsic property of the emitter which is driven by the heavy-hole light-hole mixing in the valence band that influences the optical transition dipole moments and the respective polarization components due to strong confinement anisotropy [2325]. In case of QDashes it is evidently related to the elongation [26], but also depends on the nanostructure cross-sectional size [27]. Undoubtedly, significantly larger DOLP value has been measured for single QDash neutral exciton on rectangular mesa, typically exhibiting an enhanced DOLP above $50 \%$.



Fig. 1. (a) Photoluminescence of linearly polarized spectra from inhomogeneous ensemble of quantum dashes and (b) for single exciton emission from processed asymmetric mesa structure. The inset in the left graph shows a SEM image of an uncapped QDash layer, and a schematic illustration of a Qdash (white) embedded in a rectangular mesa (blue rectangle).

In order to verify the origin of the increased DOLP for the neutral exciton, we made further experimental steps and looked at a correlation between the structure of the surrounding dielectric medium and the exciton emission. While enhancement of the [1-10]-polarized exciton transition is consistent with the longer axis of rectangular mesa, we applied two different geometries in the same experiment. One is the symmetric squared mesa of $500 \mathrm{~nm} \times 500 \mathrm{~nm}$ size and the second is the same rectangle as in Fig. 1, aligned perpendicular to the [1-10] crystallographic direction. The results are presented in Fig. 2 


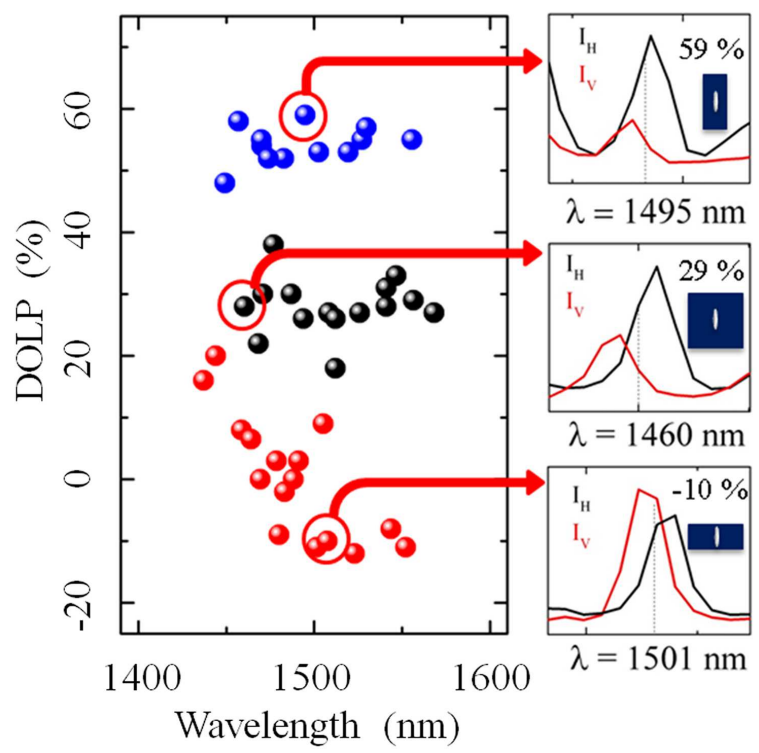

Fig. 2. DOLP of single quantum dash exciton from various mesa geometries oriented along the QDash elongation (blue), perpendicular (red) and square (black). On the right hand side exemplary excitonic microphotoluminescence spectra are presented.

with a number of measured values of excitonic DOLP. The different geometries have a clear impact on the polarization anisotropy. One can see that a squared mesa does not significantly modify the emission of the exciton and the DOLP level is similar to the average value obtained from the ensemble emission. Interestingly, the impact of a perpendicular rectangle on the emitter is as significant as the parallel one $[1-10]$, giving rise to a reduction of the intrinsic DOLP or even reversing its sign. The available tuning range for the QDash DOLP by changing the geometry in this way approaches $70 \%$ in total. A dispersion of $10 \%$ in each case may be attributed to the possible size irregularities resulting from the etching process of dielectric medium but also from the random emitter position on the sample, both of which influence the light-matter interaction.

In order to evaluate if further DOLP increase is possible, aiming at $100 \%$ and at a continuous tuning by the dielectric medium geometry, we employed a numerical model of electromagnetic field distribution inside a dielectric mesa structure using finite element method (FEM) implemented in COMSOL Multiphysics software (Wave Optics module) [28]. The concept of this simulation method assumes reversibility of the light wave propagation on emitter-detector distance. Such an approach allows us to avoid simulating emitted wave components which do not reach the detector. More specifically, we back-propagate linearly polarized plane waves from the detector plane and then we search for resultant scattered field in the emitter plane [29]. The simulation that generates the scattered field is realized in two steps. First, we neglect the mesa in simulation box which consists of free-space (vacuum) and dielectric substrate. The electromagnetic field distribution is calculated using periodic boundary conditions on a substrate plane under illumination by a polarized plane wave. The incident field acts as a background field when the mesa is introduced in the subsequent recalculation of a total field. We encapsulated the simulation box in perfectly matched layers (PMLs) boundary condition to account for proper calculation of scattered waves. In this model one can calculate the influence of mesa parameters (shape, dimensions, material) on the available states of the electromagnetic field. The resultant electric field vector in the middle of the emitter plane can be characterized by the optical polarization anisotropy

$$
\xi=\frac{\left|\boldsymbol{E}_{l}\right|^{2}-\left|\boldsymbol{E}_{t}\right|^{2}}{\left|\boldsymbol{E}_{l}\right|^{2}+\left|\boldsymbol{E}_{t}\right|^{2}},
$$

where $\left|\boldsymbol{E}_{\lambda}\right|$ is the electric field amplitude in the center of the mesa for the outgoing polarization $\lambda$ and the polarization axes labeled as $l, t$ refer to the directions along and across the elongation of the QDash, respectively.

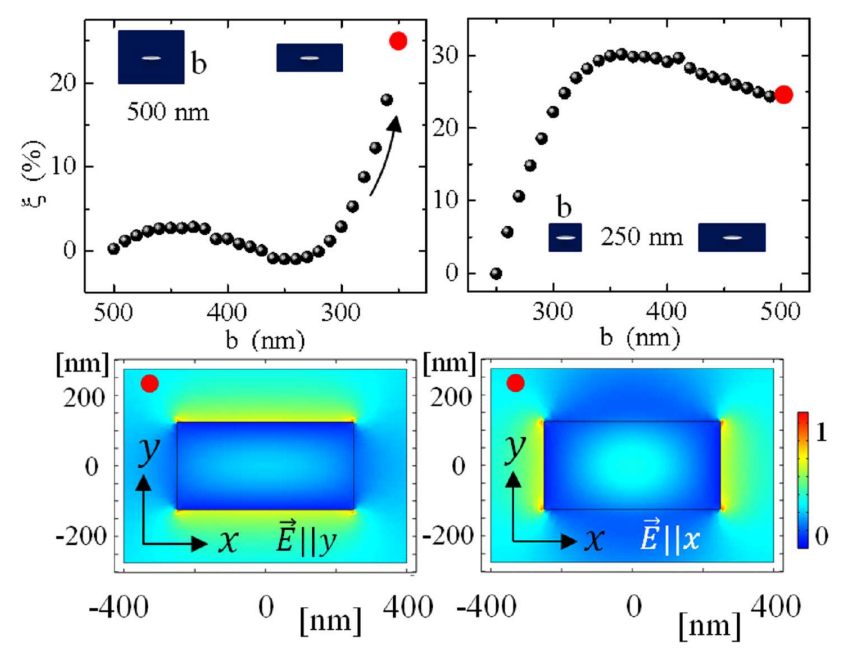

Fig. 3. Calculated optical degree of linear polarization $\xi$ for various sample geometries. The incident wavelength is $1550 \mathrm{~nm}$. The upper left figure shows $\xi$ for a $500 \mathrm{~nm}$ long mesa with different width, the upper right the case of a $250 \mathrm{~nm}$ wide mesa with different length. The two lower figures show the electromagnetic field distribution of a $250 \mathrm{~nm} \times 500 \mathrm{~nm}$ mesa structure with $\xi=25 \%$ for incident plane wave polarized along [110] (left) and [1-10] (right).

The results of the optical simulation are then utilized in the calculation of the oscillator strength of the fundamental transition by the Fermi golden rule within the multiband $k \cdot p$ model of the emitter, including valence band mixing related to the intrinsic degree of linear polarization [26]. The calculated intensity of light emitted into polarizations is

$$
I_{\lambda} \sim\left|\boldsymbol{E}_{\lambda}\right|^{2}\left|\hat{e}_{\lambda} \boldsymbol{d}_{0}\right|
$$

where $\hat{e}_{\lambda}$ is the unity vector defined as $\hat{e}_{l}=\left(\hat{e}_{x}-\hat{e}_{y}\right) / \sqrt{2}$, $\hat{e}_{t}=\left(\hat{e}_{x}+\hat{e}_{y}\right) / \sqrt{2}, x, y$ are the in-plane crystallographic 
directions [100] $([010])$, and $\left|\boldsymbol{d}_{0}\right|$ is the interband dipole moment for a nominally heavy hole transition including a light hole admixture of amplitude $\epsilon$ [26]. The expression for the DOLP of a QDash influenced by a dielectric environment is then

$$
\operatorname{DOLP}=\frac{\left|I_{l}\right|^{2}-\left|I_{t}\right|^{2}}{\left|I_{l}\right|^{2}+\left|I_{t}\right|^{2}}=\xi+\frac{2}{\sqrt{3}} \epsilon\left(1-\xi^{2}\right) .
$$

Figure 3 shows the calculated dependence of $\xi$ on various geometries of a dielectric square mesa. As expected, for increasing asymmetry of the mesa by narrowing or length, the polarization anisotropy increases, saturating at about $30 \%$ in degree of linear polarization. For the experimentally studied mesa of the in-plane aspect ratio of 2 we obtain $25 \%$. Using Eq. (3) with $\epsilon=0.21$ corresponding to the QDash DOLP of $29 \%$ in an unstructured environment we get a total DOLP of $46 \%$. Considering the higher DOLP from the experiment, we suppose that a slight deviation could be associated with some imperfections regarding etching process of the mesa or more likely with the off-center position of the QDash.

\section{Conclusions}

In this work we presented the analysis of polarization anisotropy of emission from a single quantum dash which is sensitive to the size and geometry of dielectric medium. Comparing results for QDashes emitting at $1.55 \mu \mathrm{m}$ for rectangular mesa with its lateral aspect ratio of 2 we found a satisfactory correspondence between the experimental DOLP of a QDash oriented along the longer edge, and the calculated value using multi-band $k \cdot p$ modeling and optical field simulations. We believe that further study will open up a route towards post-growth control and possible enhancement of polarization anisotropy of the emitter leading to increased efficiency of linearly polarized single photon sources.

\section{Acknowledgments}

This research was supported by the Polish Ministry of Science and Higher Education/the National Science Center Grant No. 2011/02/A/ST3/00152. The experiments have partially been performed within the laboratory infrastructure financed by the Polish Ministry of Science and Higher Education Grant No. 6167/IA/119/2012.

\section{References}

[1] E. Knill, R. Laflamme, G.J. Milburn, Nature 409, 46 (2001).

[2] N. Gisin, G. Ribordy, W. Tittel, H. Zbinden, Rev. Mod. Phys. 74, 145 (2002).

[3] C.H. Bennet, G. Brassard, in: Proc. IEEE Int. Conf. Comput. Syst. Signal Process., Bangalore 1984, p. 175.

[4] M. Williamson, V. Vedral, J. Appl. Opt. 50, 1989 (2002).
[5] A. Ugur, S. Kremling, F. Hatami, S. Höfling, L. Worschech, A. Forchel, W. Ted Masselink, Appl. Phys. Lett. 100, 023116 (2012).

[6] R. Thompson, R. Stevenson, A.J. Shields, I. Farrer, C. Lobo, D.A. Ritchie, M. Leadbeater, M. Pepper, Phys. Rev. B 64, 201302 (2001).

[7] E. Dusanowski, A. Golnik, M. Syperek, M. Nawrocki, G. Sęk, J. Misiewicz, T.W. Schlereth, C. Schneider, S. Höfling, M. Kamp, A. Forchel, Appl. Phys. Lett. 101, 103108 (2012).

[8] Ł. Dusanowski, M. Syperek, P. Mrowiński, W. RudnoRudziński, J. Misiewicz, A. Somers, S. Höfling, M. Kamp, J.P. Reithmaier, G. Sęk, Appl. Phys. Lett. 105, 021909 (2014).

[9] A. Lundskog, C.-W. Hsu, K.F. Karlsson, S. Amloy, D. Nilsson, U. Forsberg, P.O. Holtz, E. Janzén, Light Sci. Appl. 3, 139 (2014).

[10] M.H.M. van Weert, N. Akopian, F. Kelkensberg, U. Perinetti, M.P. van Kouwen, J.G. Rivas, M.T. Borgström, R.E. Algra, M.A. Verheijen, E.P.A.M. Bakkers, L.P. Kouwenhoven, V. Zwiller, Small 5, 2134 (2009).

[11] J. Wang, M.S. Gudiksen, X. Duan, Y. Cui, C.M. Lieber, Science 293, 1455 (2001).

[12] A.P. Foster, J.P. Bradley, K. Gardner, A.B. Krysa, B. Royall, M.S. Skolnick, L.R. Wilson, Nano Lett. 15, 1559 (2015).

[13] P. Stepanov, A. Delga, X. Zang, E. Dupuy, E. Peinke, P. Lalanne, J. Claudon, Appl. Phys. Lett. 106 , 041112 (2015).

[14] E. Gallardo, L.J. Martínez, A.K. Nowak, H.P. van der Meulen, J.M. Calleja, C. Tejedor, I. Prieto, D. Granados, A.G. Taboada, J.M. García, P.A. Postigo, Opt. Express 18, 13301 (2010).

[15] M.T. Rakher, N.G. Stoltz, L.A. Coldren, P.M. Petroff, D. Bouwmeester, Appl. Phys. Lett. 93, 091118 (2008).

[16] D. Unitt, A.J. Bennett, P. Atkinson, D.A. Ritchie, A.J. Shields, Phys. Rev. B 72, 033318 (2005).

[17] J. Yuan, H. Wang, R.P.J. Van Veldhoven, J. Wang, T. De Vries, B. Smalbrugge, C.Y. Jin, P. Nouwens, E.J. Geluk, A.Y. Silov, R. Nötzel, Appl. Phys. Lett. 98, 201904 (2011).

[18] Ł. Dusanowski, M. Syperek, W. Rudno-Rudziński, P. Mrowiński, G. Sęk, J. Misiewicz, A. Somers, J.P. Reithmaier, S. Höfling, A. Forchel, Appl. Phys. Lett. 103, 253113 (2013).

[19] P. Mrowiński, A. Musiał, A. Maryński, M. Syperek, J. Misiewicz, A. Somers, J.P. Reithmaier, S. Höfling, G. Sęk, Appl. Phys. Lett. 106, 053114 (2015).

[20] A. Sauerwald, T. Kummell, G. Bacher, A. Somers, R. Schwertberger, J.P. Reithmaier, A. Forchel, Appl. Phys. Lett. 86, 253112 (2005).

[21] G. Sek, P. Podemski, A. Musiał, J. Misiewicz, S. Hein, S. Höfling, A. Forchel, J. Appl. Phys. 105, 086104 (2009).

[22] P. Mrowiński, A. Musiał, G. Sęk, J. Misiewicz, S. Höfling, A. Somers, S. Hein, A. Forchel, Acta Phys. Pol. A 124, 801 (2013).

[23] S. Ohno, S. Adachi, R. Kaji, S. Muto, H. Sasakura, Appl. Phys. Lett. 98, 161912 (2011). 
[24] C. Tonin, R. Hostein, V. Voliotis, R. Grousson, A. Lemaitre, A. Martinez, Phys. Rev. B Condens. Matter Mater. Phys. 85, 155303 (2012).

[25] H. Tahara, Y. Ogawa, F. Minami, K. Akahane, M. Sasaki, Phys. Rev. B Condens. Matter Mater. Phys. 87, 035304 (2013).

[26] A. Musiał, P. Kaczmarkiewicz, G. Sęk, P. Podemski, P. Machnikowski, J. Misiewicz, S. Hein, S. Höfling, A. Forchel, Phys. Rev. B Condens. Matter Mater. Phys. 85, 035314 (2012).
[27] A. Musiał, P. Podemski, G. Sęk, P. Kaczmarkiewicz, J. Andrzejewski, P. Machnikowski, J. Misiewicz, S. Hein, A. Somers, S. Höfling, J.P. Reithmaier, A. Forchel, Semicond. Sci. Technol. 27, 105022 (2012).

[28] J.M. Jin, The Finite Element Method in Electromagnetics, 2nd ed., Wiley, New York 2002.

[29] Scatterer on Substrate, COMSOL tutorial. 